\title{
The role of the CDCA gene family in ovarian cancer
}

\author{
Chongxiang Chen ${ }^{1,2 \#}$, Siliang Chen ${ }^{3 \#}$, Ma Luo ${ }^{4 \#}$, Honghong Yan ${ }^{2}$, Lanlan Pang ${ }^{5}$, Chaoyang Zhu ${ }^{5}$, \\ Weiyan Tan ${ }^{5}$ Qingyu Zhao ${ }^{2}$, Jielan Lai ${ }^{6}$, Huan $\mathrm{Li}^{2}$
}

${ }^{1}$ Guangzhou Institute of Respiratory Diseases, State Key Laboratory of Respiratory Disease, the First Affiliated Hospital of Guangzhou Medical University, Guangzhou 510120, China; ${ }^{2}$ Department of Intensive Care Unit, Sun Yat-sen University Cancer Center, State Key Laboratory of Oncology in South China, Collaborative Innovation Center for Cancer Medicine, Guangzhou 510060, China; ${ }^{3}$ Department of Hematology, Sun Yat-sen University Cancer Center, State Key Laboratory of Oncology in South China, Collaborative Innovation Center for Cancer Medicine, Guangzhou 510060, China; ${ }^{4}$ Department of Interventional Radiology, Sun Yat-sen University Cancer Center, State Key Laboratory of Oncology in South China, Collaborative Innovation Center for Cancer Medicine, Guangzhou 510060, China; ${ }^{5}$ Zhongshan School of Medicine, Sun Yat-sen University, Guangzhou 510080, China; ${ }^{6}$ Department of Anesthesiology, State Key Laboratory of Oncology in South China, Collaborative Innovation Center for Cancer Medicine, Sun Yat-sen University Cancer Center, Guangzhou, Guangdong 510060, China

Contributions: (I) Conception and design: H Li, J Lai; (II) Administrative support: H Li; (III) Provision of study materials or patients: C Chen, S Chen; (IV) Collection and assembly of data: C Chen, S Chen, M Luo, H Yan, L Pang; (V) Data analysis and interpretation: H Li, J Lai, C Chen, S Chen, M Luo; (VI) Manuscript writing: All authors; (VII) Final approval of manuscript: All authors.

\#These authors contributed equally to this work.

Correspondence to: Huan Li. State Key Laboratory of Oncology in South China and Department of Intensive Care Unit, Sun Yat-sen University Cancer Center, 651 Dongfeng Dong Road, Guangzhou 510060, China. Email: lihuan@sysucc.org.cn; Jielan Lai. State Key Laboratory of Oncology in South China and Department of Anesthesiology, Sun Yat-sen University Cancer Center, 651 Dongfeng Dong Road, Guangzhou 510060, China. Email: laijl@sysucc.org.cn.

Background: Ovarian cancer is a frequently-occurring reproductive system malignancy in females, which leads to an annual of over 100 thousand deaths worldwide.

Methods: The electronic databases, including GEPIA, ONCOMINE, Metascape, and Kaplan-Meier Plotter, were used to examine both survival and transcriptional data regarding the cell division cycle associated $(C D C A)$ gene family among ovarian cancer patients.

Results: All CDCA genes expression levels were up-regulated in ovarian cancer tissues relative to those in non-carcinoma ovarian counterparts. Besides, $C D C A 5 / 7$ expression levels were related to the late tumor stage. In addition, the Kaplan-Meier Plotter database was employed to carry out survival analysis, which suggested that ovarian cancer patients with increased $C D C A 2 / 3 / 5 / 7$ expression levels had poor overall survival (OS) $(\mathrm{P}<0.05)$. Moreover, ovarian cancer patients that had up-regulated mRNA expression levels of $C D C A 2 / 5 / 8$ had markedly reduced progression-free survival (PFS) $(\mathrm{P}<0.05)$; and up-regulated $C D C A 4$ expression showed remarkable association with reduced post-progression survival (PPS) $(\mathrm{P}<0.05)$. Additionally, the following processes were affected by CDCA genes alterations, including R-HAS-2500257: resolution of sister chromatid cohesion; GO:0051301: cell division; CORUM: 1118: Chromosomal passenger complex (CPC, including CDCA8, INCENP, AURKB and BIRC5); CORUM: 127: NDC80 kinetochore complex; M129: PID PLK1 pathway; and GO: 0007080: mitotic metaphase plate congression, all of which were subjected to marked regulation since the alterations affected $C D C A$ genes.

Conclusions: Up-regulated $C D C A$ gene expression in ovarian cancer tissues probably played a crucial part in the occurrence of ovarian cancer. The up-regulated $C D C A 2 / 3 / 5 / 7$ expression levels were used as the potential prognostic markers to improve the poor ovarian cancer survival and prognostic accuracy. Moreover, $C D C A$ genes probably exerted their functions in tumorigenesis through the PLK1 pathway.

Keywords: Ovarian cancer; cell division cycle associated $(C D C A)$; prognosis

Submitted Oct 11, 2019. Accepted for publication Jan 03, 2020.

doi: $10.21037 /$ atm.2020.01.99

View this article at: http://dx.doi.org/10.21037/atm.2020.01.99 


\section{Introduction}

Ovarian cancer is a frequently-occurring reproductive system malignancy in females, which is estimated to cause an annual of over 100 thousand deaths worldwide (1). Disappointedly, more than $70 \%$ patients with ovarian cancer are diagnosed at the advanced stage due to the lack of efficient diagnostic method and early typical clinical symptom (2). Although treatments (such as surgery and targeted therapeutics) have been improved, they cannot achieve satisfactory progression-free survival (PFS) among patients with ovarian cancer; besides, the subsequent treatment for relapsed ovarian cancer is still encountered with great challenges (3). Additionally, the 5-year survival of ovarian cancer patients is only about $46.5 \%$ (4). Consequently, it is necessary to examine the underlying mechanisms regarding ovarian cancer tumorigenesis as well as progression and to identify the related tumor biomarkers that have high specificity and sensitivity. Nowadays, several molecular tests have been utilized prior to treatment for the risk screening of ovarian cancer, including BRCA1/2 mutations, microsatellite instability, and homologous recombination pathway genes. In addition, bevacizumab and Olaparib have been recommended by the National Comprehensive Cancer Network (NCCN) guideline for the treatment of ovarian cancer (5). All in all, BRCA1/2 mutations are not only used as the targets of agents like Olaparib (6), Rucaparib (7), and Niraparib (8), but also act as the high risk factors, which contribute to early screening (9-13). In patients with high risk factors (like $B R C A$ mutation, family history), and cancer antigen 125 (CA$125)$, ultrasound is adopted to identify patients with ovarian cancer (14). More efforts should be made to search for more beneficial genes to predict cancer occurrence or targeted therapy. There are 8 members in the $C D C A$ protein family, namely, $C D C A 1-8$. Cell division plays an important role in the life process. It has been suggested in numerous studies that any dysregulation in the process of cell division may lead to malignancy (15-17). CDCA1 is known to be a member of a highly conserved $\mathrm{Ndc} 80$ complex that plays a crucial role in spindle checkpoint signaling (18). CDCA2 plays a role in modulating response of DNA injury within cell cycle, which is achieved through binding onto protein phosphatase $1 \gamma$ (PP1 $\gamma)(19,20)$. CDCA3 functions to modulate the progression of cell cycle, and the expression level is regulated via protein degradation and transcription at G1 phase in cell cycle (21). Moreover, CDCA4 can regulate the cell cycle, which is related to transition of G1/S phase (22) and regulates the expression of p53 (23). CDCA5 serves as a primary regulatory factor for the sister-chromatid separation and cohesion (24). CDCA6, also named as $C B X 2$, is a gene whose protein product forms part of the PRC1, a multi-protein complex that modifies histones (25). In the undifferentiated hematopoietic populations, $C D C A 7$ can be triggered in the precursors of hematopoietic stem cells within murine embryo, which can be maintained afterwards. Besides, CDCA 8 plays an essential role in regulating mitosis (26).

Nowadays, several studies on using some $C D C A$ family genes as the prognostic factors have raised our attentions (27-29). However, there is little systematical analysis on the role of $C D C A$ gene family in patients with ovarian cancer. The current research aimed to systematically evaluate the association of $C D C A$ genes expression with ovarian cancer survival. Typically, the mRNA expression of CDCA genes was detected in both normal and ovarian cancer tissues. Then, the significance of all CDCA family members in predicting the prognosis for ovarian cancer was analyzed based on the Kaplan-Meier plotter database, and later the gene-gene interaction network was constructed for $C D C A$ genes to examine the underlying mechanisms of action. This study explored the $C D C A$ genes clinical value, so as to provide a certain theoretical foundation for making early diagnosis, prognosis evaluation, and specific treatment for ovarian cancer.

\section{Methods}

Each dataset used in this study was searched based on the published literature. The clinical tumor samples were collected during the first surgery, the normal specimens belonged to the same patients, and the threshold used to define low and high expression was $50 \%$ median. Additionally, the included literature datasets (TCGA datasets and GEO datasets) used for calculating KaplanMeier survival in Kaplan-Meier Plotter (www. Kmplot.com) are shown in Table S1.

\section{ONCOMINE analyses}

The transcription levels of $C D C A$ genes among various cancer types were examined based on the online cancer microarray database, namely, the ONCOMINE gene expression array dataset (www.oncomine.org/). Moreover, $C D C A$ s mRNA expression was compared between the clinical tumor samples and normal specimens. The $\mathrm{P}$ value 
was generated by Students' $t$-test, and the threshold foldchange and $\mathrm{P}$ value were set at 2 and 0.01 , respectively.

\section{The Gene Expression Profiling Interactive Analysis (GEPIA) dataset}

GEPIA, the newly designed interactive web server, was used to analyze RNA sequencing materials based on the GTEx and TCGA projects by the use of the normalized processing pipeline. Typically, GEPIA allows to offer the differential expression analyses on normal and tumor tissues, as well as the access to profiling of cancer type and pathological stage, analysis of patient survival, detection of similar gene, as well as dimensionality reduction and correlation analyses.

\section{Database Kaplan-Meier plotter}

Kaplan-Meier Plotter (www. Kmplot.com), the online database, was used to assess the prognostic significance of the mRNA expression levels of $C D C A$ genes. For analyzing the ovarian cancer patient overall survival (OS), PFS, as well as the post progression survival (PPS), all specimens were divided as 2 groups according to the $50 \%$ median expression level (namely, low and high expression).

The baseline clinical data of included literature datasets in Kaplan-Meier Plotter for analyzing were collected, and then these data were included to perform bioinformatic Cox regression analysis for OS by $\mathrm{R}$ software (version 3.6.1). Later, the Kaplan-Meier survival plot was used for evaluation on the basis of hazard ratio (HR) and the corresponding $95 \%$ confidence intervals (CI), as well as the $\log$-rank $\mathrm{P}$ value. The Kaplan-Meier plots were obtained through the $C D C A$ s JetSet best probe set alone, where the number at risk was suggested under the major plot.

\section{Bioinformatic analyses and functional enrichment}

The online database metascape (http://metascape.org) has integrated more than 40 bioinformatic knowledge bases, which enables to extract rich annotations, identify the enriched pathways, and construct the protein-protein interaction (PPI) (30) network based on the lists of protein and gene identifiers. The $C D C A$ genes were analyzed using the Kyoto Encyclopedia of Genes and Genomes (KEGG) and Gene Ontology (GO) approaches of Metascape, so as to search for those linked genes with the highest alteration frequency.

\section{Results}

Six $C D C A$ factors are identified within the mammalian cells. In this study, the ONCOMINE databases were used to compare the $C D C A$ gene transcriptional data between tumor tissues and normal specimens (Figure 1). According to our findings, CDCA2/3/4/5/7/8 expression remarkably increased among ovarian cancer patients derived from many databases. For $C D C A 2$, the result from Yoshihara's dataset showed that the fold change was 26.877 (31). TCGA dataset showed a fold change of 4.847 in CDCA3. In addition, TCGA dataset demonstrated that the fold change was 2.213 in $C D C A 4$. For $C D C A 5$, Yoshihara's dataset revealed a fold change of 12.508 (31). For CDCA7, the results by Lu's database suggested that the fold change was 2.646 (32), which were 8.261 as suggested by Yoshihara's dataset (31). For CDCA8, Yoshihara's dataset showed a fold change of 20.335, which was 3.705 in TCGA dataset and 2.036 as suggested by the Bonome's dataset (33) (Table 1).

\section{Associations of CDCAs mRNA expression with clinicopathological variables in ovarian cancer patients}

The GEPIA dataset (http://gepia.cancer-pku.cn/) was performed to compare $C D C A \mathrm{~s}$ mRNA expression in ovarian cancer tissues with that in normal ovarian tissues. According to our findings, the CDCA2/3/4/5/7/8 expression levels (tumor sample: $\mathrm{n}=426$ vs. normal sample: $\mathrm{n}=88$ ) were upregulated within ovarian cancer tissues relative to those within normal tissues. (Figures 2,3) Also, the association between $C D C A$ genes expression and the ovarian cancer stage was analyzed. There were significant differences in the expression levels of all CDCA genes (Figure 4).

\section{Relationship between elevated CDCA 2/3/4/5/7/8 mRNA expression and dismal prognosis for ovarian cancer cases}

The Kaplan-Meier Plotter (www.Kmplot.com) was utilized to examine the relationship between CDCAs mRNA expression and ovarian cancer patients' survival by the use of the public datasets. The clinical baseline data of datasets included in Kaplan-Meier Plotter for analysis are displayed in Table S1. The multivariate analysis (using GSE 18920, GSE 26193, GSE 30161, GSE 63888) indicated that stage and CDCA7 were the independent risk factors (Table S2, Figure S1). Based on the long-rank test and Kaplan-Meier curve analyses, it was found that the elevated $C D C A 2 / 3 / 5 / 7$ 


\begin{tabular}{|r|}
\hline Analysis Type by Cancer \\
\hline Bladder Cancer \\
\hline Brain and CNS Cancer \\
\hline Breast Cancer \\
Cervical Cancer \\
\hline Colorectal Cancer \\
\hline Esophageal Cancer \\
\hline Gastric Cancer \\
\hline Head and Neck Cancer \\
\hline Kidney Cancer \\
\hline Leuk emia \\
\hline Liver Cancer \\
Lung Cancer \\
Lymphoma \\
\hline Melanoma \\
\hline Myeloma \\
\hline Other Cancer \\
\hline Ovarian Cancer \\
\hline Pancreatic Cancer \\
\hline Prostate Cancer \\
Sarcoma \\
\hline Total Unique Analyses \\
\hline
\end{tabular}

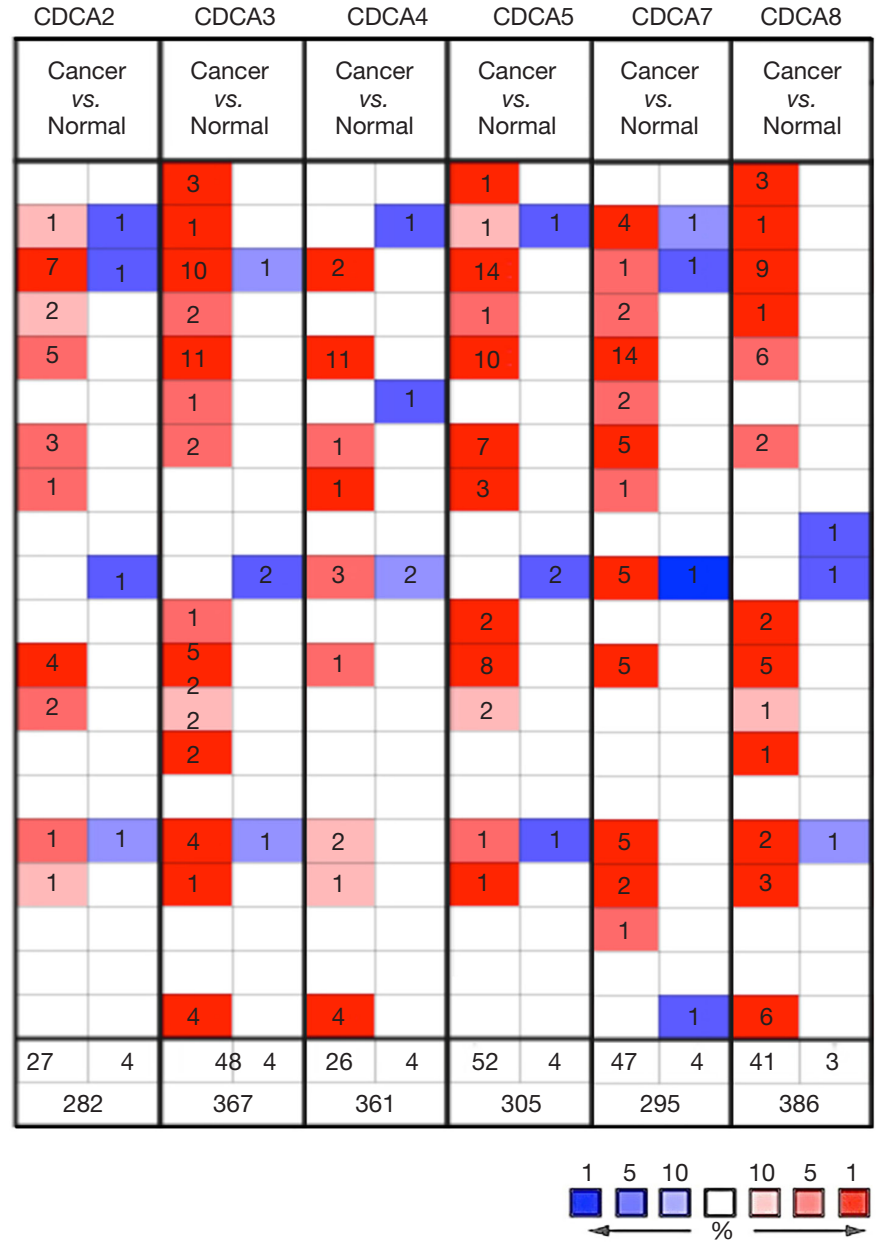

Figure 1 CDCAs expression at the transcription level among various cancer types (the ONCOMINE). (Color red means high expression level in cancer sample, and color blue means low expression level in cancer sample).

Table 1 Remarkable CDCA expression changes at transcription level between ovarian cancer tissues and the non-carcinoma counterparts (Oncomine Database)

\begin{tabular}{|c|c|c|c|c|c|}
\hline $\begin{array}{l}\text { CDCA } \\
\text { genes }\end{array}$ & Type of ovarian cancer versus normal lung tissue & Fold change & $P$ value & $t$-test & Source and/or reference \\
\hline CDCA2 & Ovarian serous adenocarcinoma & 26.877 & $1.07 \mathrm{E}-5$ & 9.218 & Yoshihara (31) \\
\hline CDCA4 & Ovarian serous cystadenocarcinoma & 2.213 & $6.40 \mathrm{E}-6$ & 9.679 & TCGA \\
\hline CDCA5 & Ovarian serous adenocarcinoma & 12.508 & $5.84 \mathrm{E}-13$ & 15.754 & Yoshihara (31) \\
\hline CDCA7 & Ovarian serous adenocarcinoma & 8.261 & $3.76 \mathrm{E}-6$ & 6.923 & Yoshihara (31) \\
\hline \multirow[t]{2}{*}{ CDCA8 } & Ovarian serous adenocarcinoma & 20.335 & $3.63 \mathrm{E}-12$ & 15.126 & Yoshihara (31) \\
\hline & Ovarian serous cystadenocarcinoma & 3.705 & $5.44 \mathrm{E}-7$ & 13.126 & TCGA \\
\hline
\end{tabular}



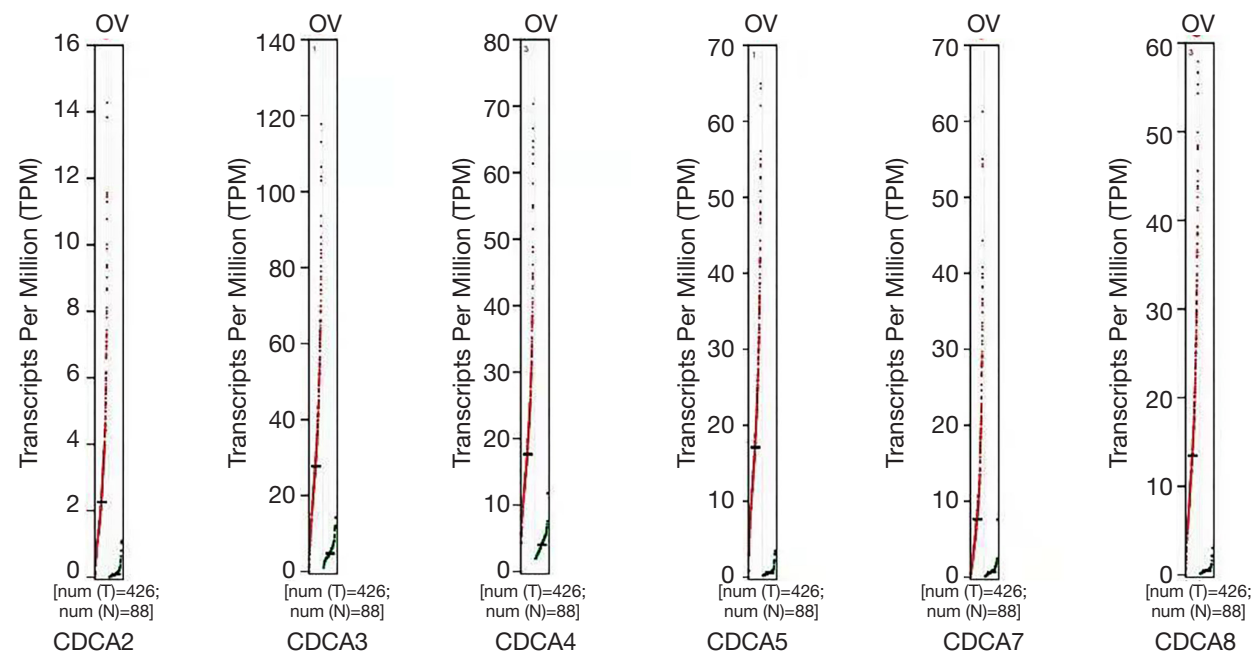

Figure 2 CDCAs expression within ovarian cancer (GEPIA).
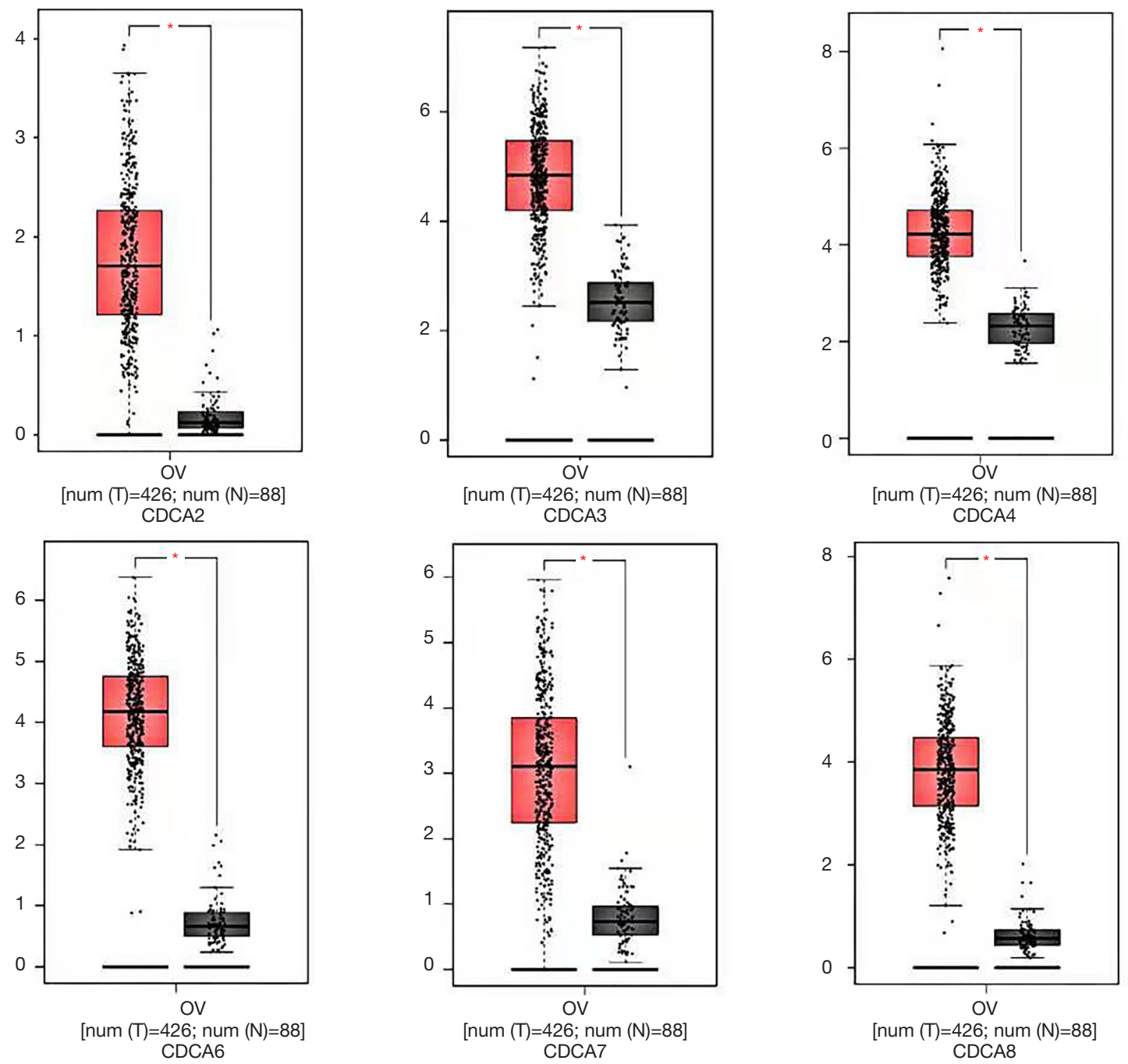

Figure 3 CDCAs expression within ovarian cancer showed as boxplot (GEPIA). ${ }^{*} \mathrm{P}<0.05$. 

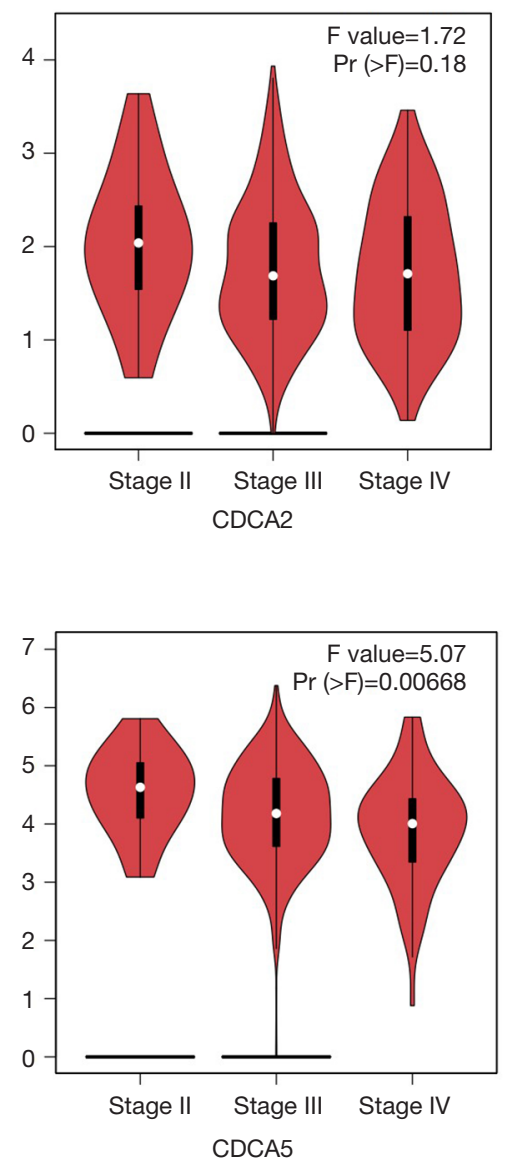
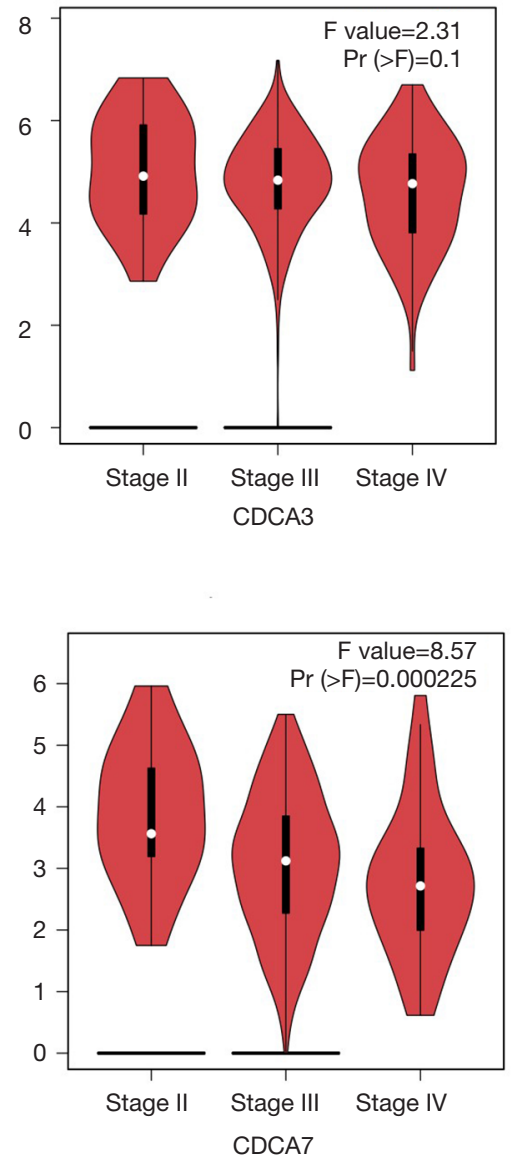
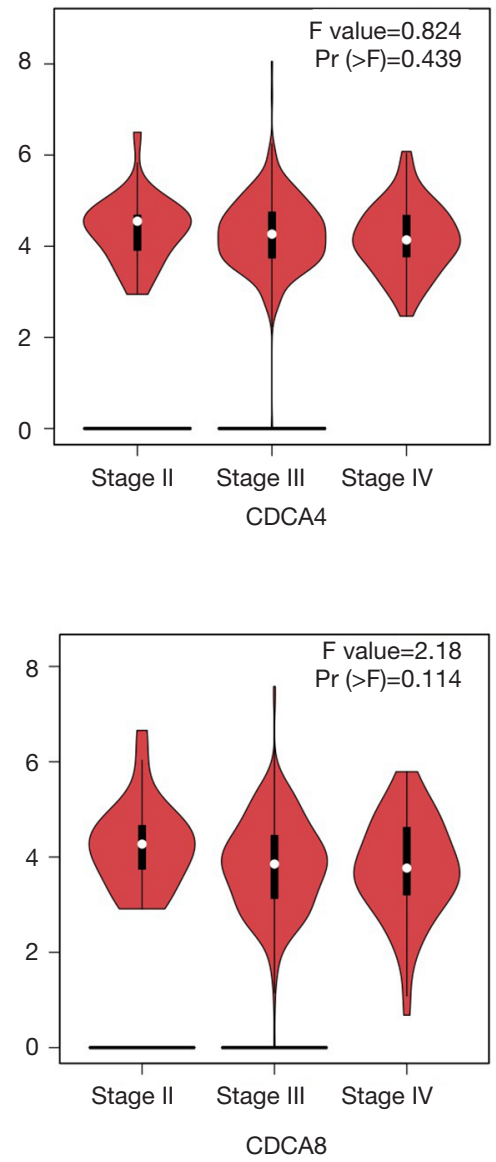

Figure 4 Relationship of CDCAs levels with cancer classification among patients with ovarian cancer (GEPIA).

expression levels showed significant relationships with poorer patient OS. Ovarian cancer patients that had upregulated $C D C A 2 / 5 / 8 \mathrm{mRNA}$ expression levels had lower $\mathrm{PFS}$, and the increased CDCA4 was only significantly associated with the lower PPS (Figure 5).

\section{Predicted functions and Pathway enrichment analyses and predicted functions of CDCA genes among ovarian cancer cases}

Genes showing co-expression with the $C D C A$ gene family were examined using the String and the Functional protein association networks. According to the results, $C D C A$ gene expression showed positive correlation with the upregulated levels of the genes shown below: $C D C A 2, N U F 2$, CDCA4, CDCA3, CDCA, CDCA5, CDCA8, CDCA7, CDC20, AURKB, CBX2, CDK1, ZWINT, BUB1, NDC80, SPC24, SPC25, BIRC5, and INCENP (Figure 6). Then, the lists of all the $C D C A$ genes expressed, together with linked genes displaying the highest alteration frequency, were compiled before they were analyzed by the KEGG and GO approaches in Metascape (Figure 7). Our results suggested that the processes below were subjected to the influence of CDCA gene alterations: R-HAS-2500257: resolution of sister chromatid cohesion; GO:0051301: cell division; CORUM: 1118: Chromosomal passenger complex (CPC, including CDCA8, INCENP, AURKB and BIRC5); CORUM: 127: NDC80 kinetochore complex; M129: PID PLK1 pathway; and GO: 0007080: mitotic metaphase plate congression.

\section{Discussion}

The guideline recommended treatments, namely, intravenous carboplatin/paclitaxel, or combined intravenous/intraperitoneal paclitaxel/cisplatin, may lead to complications like leukopenia, infection, renal toxicity, and neurotoxicity $(34,35)$. Nowadays, BRCA1/2 mutations 

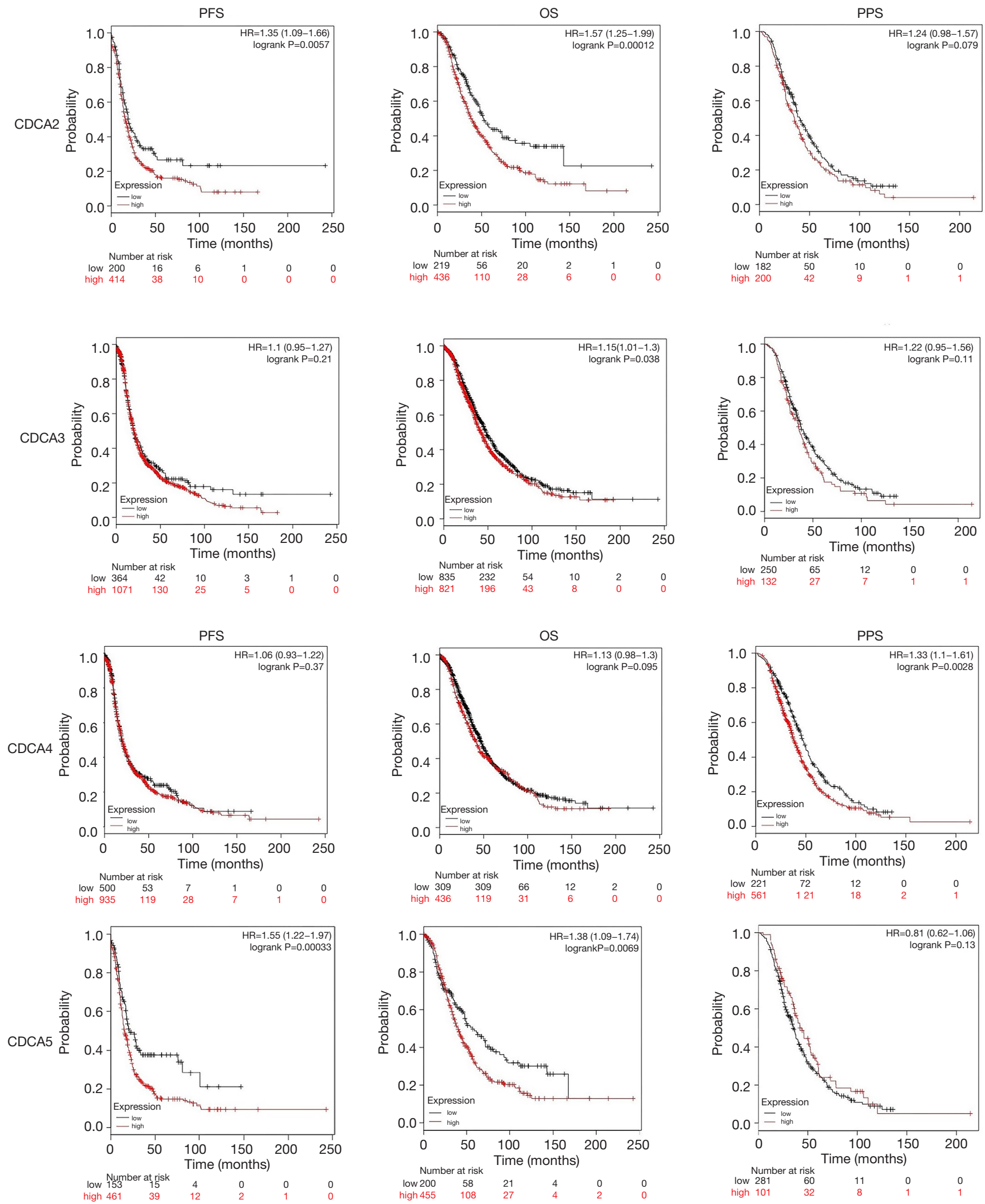

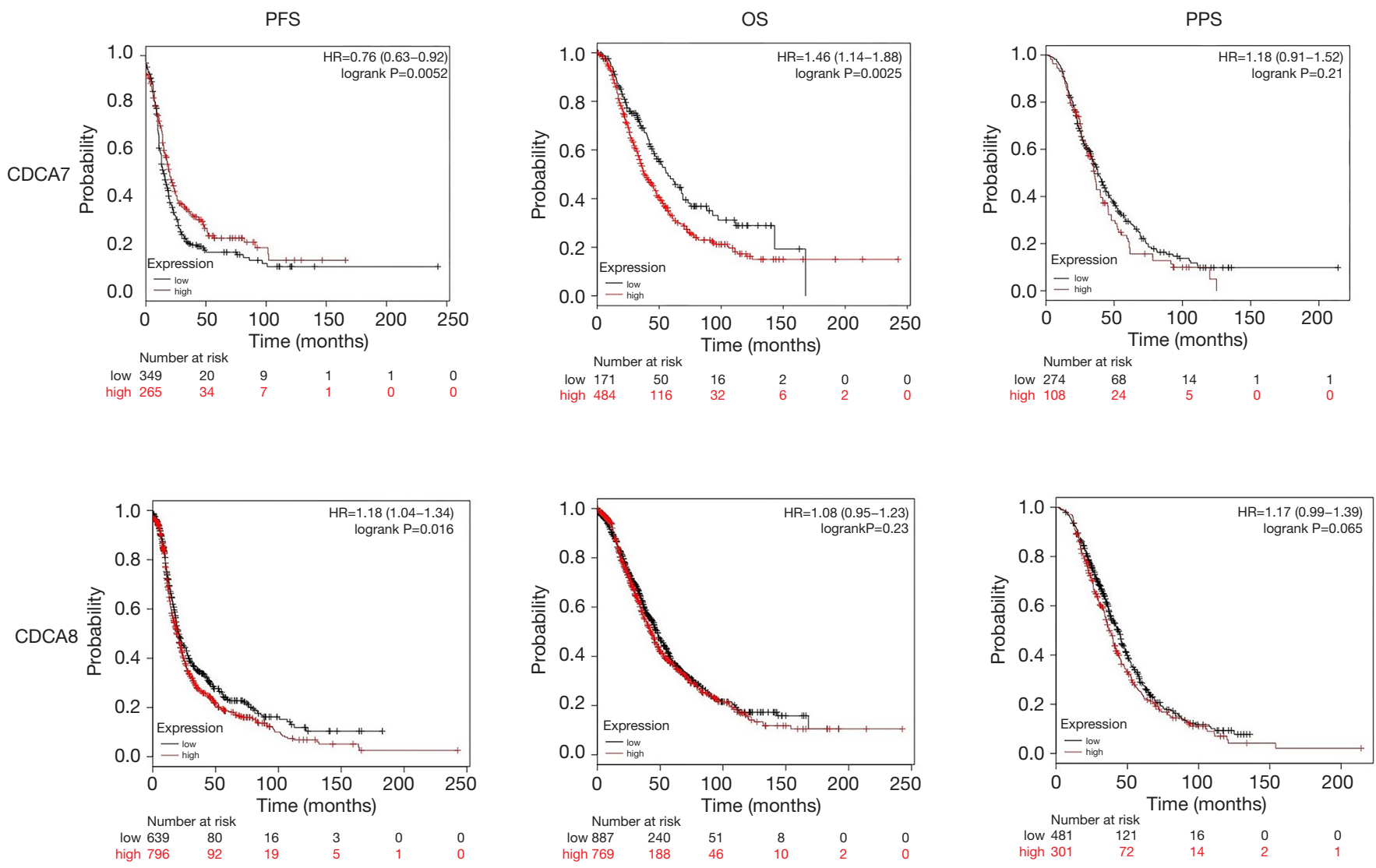

Figure 5 Significance of CDCAs mRNA expression in predicting the prognosis for patients with ovarian cancer (Kaplan-Meier plotter).

have been tested for potential therapeutics, which result in less adverse effects. Therefore, in our opinion, $C D C A$ genes probably could act as the effective therapeutics, which exert similar functions as $B R C A 1 / B R C A 2$. Our findings suggested that $C D C A$ genes expression levels in cancer patients were different from those in normal people. It is difficult to carry out early screening among patients with ovarian cancer, as suggested by our results discussing the different $C D C A$ genes expression levels at different stages, but stage 1 ovarian cancer data are lacking.

CDCA2 can regulate $\mathrm{H} 3$ (the primary mitotic histone) phosphorylation depending on PP1 (36). Our results suggested that $C D C A 2$ expression levels in ovarian cancer tissues were up-regulated compared with those in noncarcinoma counterparts. Additionally, the CDCA2 expression level was not correlated with cancer classification among ovarian cancer patients. The up-regulated $C D C A 2$ expression level showed a significant correlation with dismal OS and PFS among ovarian cancer patients.

$C D C A 3$ over-expression may be potentially related to the G1 arrest in the cell cycle, which serves as the crucial cell division checkpoint, leading to the cascade reactions that potentially result in cancer development. $C D C A 3$ is suggested to be the possible breast cancer target. $C D C A 3$ plays a role in triggering several types of cancer [such as liver cancer (37), as well as oral squamous cell cancer (38)]. It was found in this study that, $C D C A 3$ expression level was up-regulated in human ovarian cancer tissues compared with that in the non-carcinoma counterparts, whereas such expression showed no association with cancer classification among ovarian cancer patients. Commonly, the high $C D C A 3$ expression level showed a significant correlation with dismal OS for all ovarian cancer cases.

CDCA4 is one of the TRIP-Br transcription cofactor family members, which is demonstrated to play an important part in adjusting transcription factor (TF) activities, including p53 and E2F1 (22,39). It is important to determine specific target gene expression profiles in certain breast cancer subtypes, so as to develop the novel treatments. According to our results, CDCA4 expression 


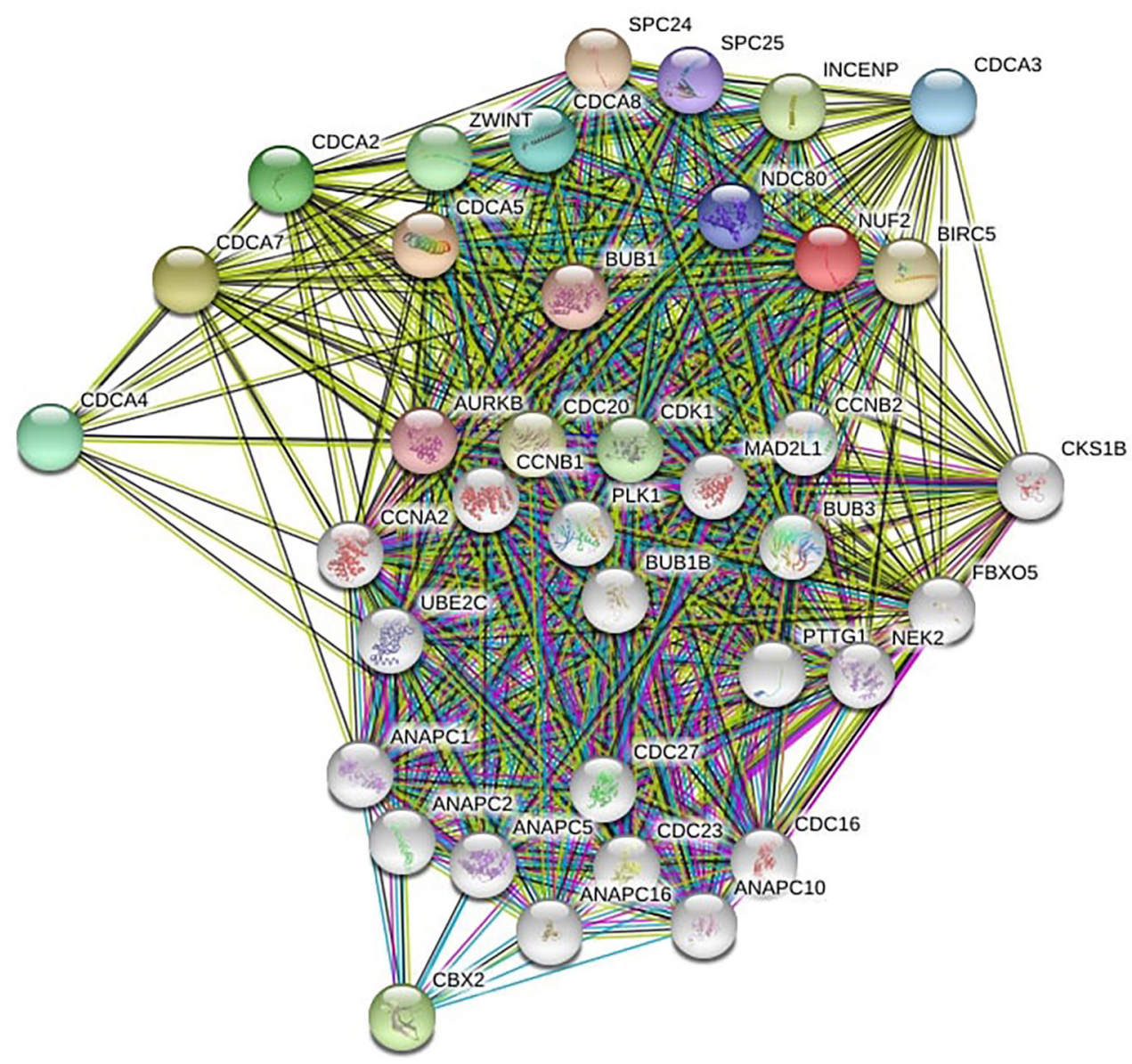

Figure 6 CDCA genes co-expression among ovarian cancer patients (String).

level was higher in human ovarian cancer tissues compared with that in non-carcinoma counterparts, and its expression level showed no correlation with cancer classification among ovarian cancer patients. Commonly, the up-regulated CDCA4 expression level showed significant correlation with the dismal PPS among all ovarian cancer patients.

As one of the oncogenes, the over-expression of $C D C A 5$ is detected in a variety of cancer types (40-42). CDCA5 exerts an important part for DNA repairs, as well as sisterchromatid cohesion and separation. The over-expression of $C D C A 5$ has been shown to be related to the dismal prognosis of lung carcinoma (24). In addition, the overexpression of $C D C A 5$ is associated with the malfunction of G1-S transition in bladder urothelial cancer (40). As found in this study, CDCA5 expression level increased in human ovarian cancer tissues compared with that in the non-carcinoma tissues, and such expression showed an association with cancer classification for ovarian cancer patients. Obviously, the high $C D C A 5$ expression level displayed distinct correlation with dismal PFS and OS among ovarian cancer cases.

In this study, $C D C A 7$ expression level was found to be up-regulated in human ovarian cancer tissues relative to that in the non-carcinoma counterparts, and such expression showed a correlation with cancer classification among ovarian cancer patients. Obviously, the high CDCA7 expression level showed significant correlation with dismal OS for ovarian cancer patients.

$C D C A 8$, which is also referred to as the Borealin/ Dasra $\mathrm{B}$, has been identified to be one of the chromosomal passenger complex (CPC) members, and it plays an indispensable role in genomic transmission in the process of cell division (43). CDCA8, which can act as a cell mitosis regulator, is demonstrated to show association with lung carcinoma after being subjected to phosphorylation at 4 sites (44). According to the previous meta-analysis, the 
A

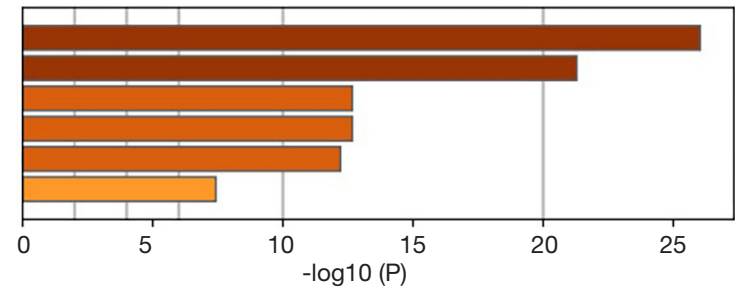

R-HSA-2500257: Resolution of Sister Chromatid Cohesion GO: 005 1301: cell division CORUM: 1118: Chromosomal passenger complex CPC (INCENP, CDCA8, BIRC5, AURKB) CORUM:127: NDC80 kinetochore complex M129: PID PLK1 PATHWAY

GO: 000 7080: mitotic meta phase plate congression

B
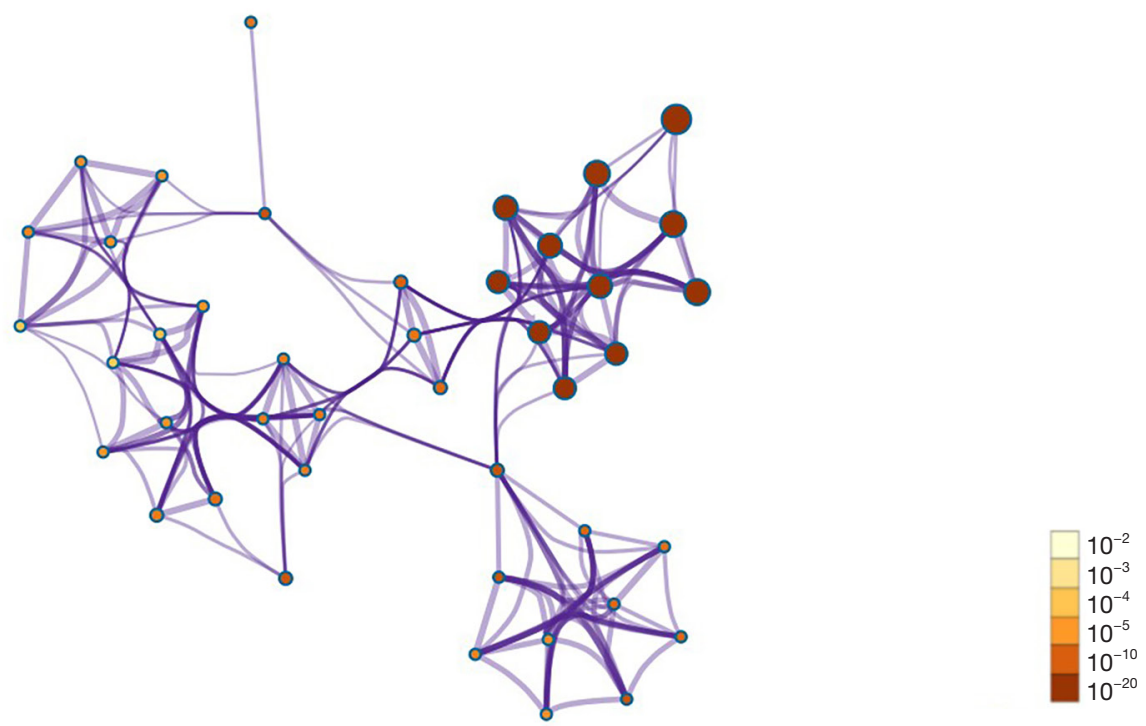

C

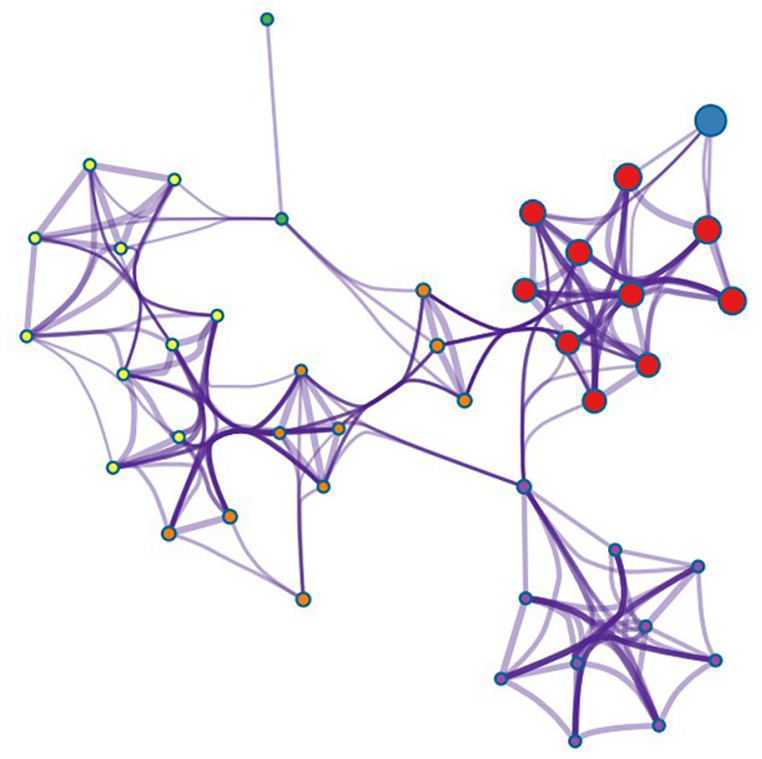

Figure 7 Functions of CDCA genes as well as those showing significant association with $C D C A$ genes alterations. (A) Heatmap of the Gene Ontology (GO) and Kyoto Encyclopedia of Genes and Genomes (KEGG) enriched terms colored by P values. (B) Network of GO and KEGG enriched terms colored by P values. (C) Network of GO and KEGG enriched terms colored by clusters. 
over-expression of $C D C A 8$ is related to the low survival of breast cancer patients (45). This study revealed that $C D C A 8$ expression level was up-regulated in human ovarian cancer tissues compared with that in non-carcinoma counterparts, and such expression showed no correlation with cancer classification among ovarian cancer patients. Obviously, the increased CDCA8 expression level displayed significant correlation with the poor PFS among ovarian cancer patients.

Besides, KEGG and GO analyses were also carried out in this study to find the correlations between $C D C A$ genes expression levels as well as linked genes of the highest alteration frequency and the prognosis for ovarian cancer. According to our results, attention should be paid to some pathways, which include: R-HAS-2500257: resolution of sister chromatid cohesion; GO: 0051301: cell division; CORUM: 1118: Chromosomal passenger complex (CPC, including CDCA8, INCENP, AURKB, and BIRC5); CORUM: 127: NDC80 kinetochore complex; M129: PID PLK1 pathway; GO: 0007080: mitotic metaphase plate congression. As a primary regulator of mitotic cell division and the DNA damage response, Polo-like kinase 1 (Plk1) is recognized as a new feasible biomarker in this area (46). PLK1 pathway has been revealed to exert certain function in patients with advanced solid malignancies (47), human hepatocellular carcinoma (HCC) $(48,49)$, and glioma (50).

Certain limitations should be noted in this study. Ovarian cancer is associated with different histological subtypes, such as serous and endometrioid (51-54), which may generate different effects in gene targeted therapy. Consequently, there is still a long way to go for this finding.

The current research systemically examines $C D C A$ genes expression levels and its prognostic significance within ovarian cancer, which sheds more light on the complexity and heterogeneity of ovarian cancer biological properties at the molecular level. This study suggests that up-regulation of $C D C A$ genes expression levels in ovarian cancer tissues probably takes a crucial role in ovarian cancer oncogenesis. Besides, the high CDCA2/3/5/7 expression levels may serve as the potential prognostic markers of the poor survival and prognostic accuracy of ovarian cancer. Moreover, $C D C A$ genes probably exert their functions in tumorigenesis through the PLK1 pathway.

\section{Acknowledgments}

Funding: This work was supported by the funds from the Medical Research Fund of Guangdong Province (No.
A2018237).

\section{Footnote}

Conflicts of Interest: The authors have no conflicts of interest to declare.

Ethical Statement: The authors are accountable for all aspects of the work in ensuring that questions related to the accuracy or integrity of any part of the work are appropriately investigated and resolved.

Open Access Statement: This is an Open Access article distributed in accordance with the Creative Commons Attribution-NonCommercial-NoDerivs 4.0 International License (CC BY-NC-ND 4.0), which permits the noncommercial replication and distribution of the article with the strict proviso that no changes or edits are made and the original work is properly cited (including links to both the formal publication through the relevant DOI and the license). See: https://creativecommons.org/licenses/by-nc-nd/4.0/.

\section{References}

1. Bray F, Ferlay J, Soerjomataram I, et al. Global cancer statistics 2018: GLOBOCAN estimates of incidence and mortality worldwide for 36 cancers in 185 countries. CA Cancer J Clin 2018;68:394-424.

2. Cortez AJ, Tudrej P, Kujawa KA, et al. Advances in ovarian cancer therapy. Cancer Chemother Pharmacol 2018;81:17-38.

3. Lheureux S, Gourley C, Vergote I, et al. Epithelial ovarian cancer. Lancet 2019;393:1240-53.

4. Jelovac D, Armstrong DK. Recent progress in the diagnosis and treatment of ovarian cancer. CA Cancer J Clin 2011;61:183-203.

5. Armstrong DK, Alvarez RD, Bakkum-Gamez JN, et al. NCCN Guidelines Insights: Ovarian Cancer, Version 1.2019. J Natl Compr Canc Netw 2019;17:896-909.

6. Pujade-Lauraine E, Ledermann JA, Selle F, et al. Olaparib tablets as maintenance therapy in patients with platinumsensitive, relapsed ovarian cancer and a BRCA1/2 mutation (SOLO2/ENGOT-Ov21): a double-blind, randomised, placebo-controlled, phase 3 trial. Lancet Oncol 2017;18:1274-84.

7. Kristeleit R, Shapiro GI, Burris HA, et al. A Phase I-II Study of the Oral PARP Inhibitor Rucaparib in Patients with Germline BRCA1/2-Mutated Ovarian Carcinoma or 
Other Solid Tumors. Clin Cancer Res 2017;23:4095-106.

8. Mirza MR, Monk BJ, Herrstedt J, et al. Niraparib Maintenance Therapy in Plati-num-Sensitive, Recurrent Ovarian Cancer. N Engl J Med 2016;375:2154-64.

9. Finch A, Beiner M, Lubinski J, et al. Salpingooophorectomy and the risk of ovarian, fallopian tube, and peritoneal cancers in women with a BRCA1 or BRCA2 Mutation. JAMA 2006;296:185-92.

10. Marchetti C, De Felice F, Palaia I, et al. Risk-reducing salpingo-oophorectomy: a me-ta-analysis on impact on ovarian cancer risk and all cause mortality in BRCA 1 and BRCA 2 mutation carriers. BMC Womens Health 2014;14:150.

11. Reitsma W, de Bock GH, Oosterwijk JC, et al. Support of the 'fallopian tube hypothesis' in a prospective series of risk-reducing salpingo-oophorectomy specimens. Eur J Cancer 2013;49:132-41.

12. Domchek SM, Friebel TM, Singer CF, et al. Association of risk-reducing surgery in BRCA1 or BRCA2 mutation carriers with cancer risk and mortality. JAMA 2010;304:967-75.

13. Rebbeck TR, Kauff ND, Domchek SM. Meta-analysis of risk reduction estimates associ-ated with risk-reducing salpingo-oophorectomy in BRCA1 or BRCA2 mutation carriers. J Natl Cancer Inst 2009;101:80-7.

14. Smith RA, Manassaram-Baptiste D, Brooks D, et al. Cancer screening in the United States, 2015: a review of current American cancer society guidelines and current issues in cancer screening. CA Cancer J Clin 2015;65:30-54.

15. Preston-Martin S, Pike MC, Ross RK, et al. Increased cell division as a cause of human cancer. Cancer Res 1990;50:7415-21.

16. Vader G, Lens SM. The Aurora kinase family in cell division and cancer. Biochim Biophys Acta 2008;1786:60-72.

17. Collins I, Garrett MD. Targeting the cell division cycle in cancer: CDK and cell cycle checkpoint kinase inhibitors. Curr Opin Pharmacol 2005;5:366-73.

18. Hayama S, Daigo Y, Kato T, et al. Activation of CDCA1KNTC2, members of centromere protein complex, involved in pulmonary carcinogenesis. Cancer Res 2006;66:10339-48.

19. Peng A, Lewellyn AL, Schiemann WP, et al. Repo-man controls a protein phosphatase 1-dependent threshold for DNA damage checkpoint activation. Curr Biol 2010;20:387-96.

20. Vagnarelli P. Repo-man at the intersection of chromatin remodelling, DNA repair, nu-clear envelope organization, and cancer progression. Adv Exp Med Biol
2014;773:401-14.

21. Yoshida K. Cell-cycle-dependent regulation of the human and mouse Tome-1 pro-moters. FEBS Lett 2005;579:1488-92.

22. Hayashi R, Goto Y, Ikeda R, et al. CDCA4 is an E2F transcription factor family-induced nuclear factor that regulates E2F-dependent transcriptional activation and cell prolifera-tion. J Biol Chem 2006;281:35633-48.

23. Tategu M, Nakagawa H, Hayashi R, et al. Transcriptional co-factor CDCA4 participates in the regulation of JUN oncogene expression. Biochimie 2008;90:1515-22.

24. Nguyen MH, Koinuma J, Ueda K, et al. Phosphorylation and activation of cell division cycle associated 5 by mitogen-activated protein kinase play a crucial role in human lung carcinogenesis. Cancer Res 2010;70:5337-47.

25. Piqué DG, Montagna C, Greally JM, et al. A novel approach to modelling transcriptional heterogeneity identifies the oncogene candidate CBX2 in invasive breast carcinoma. Br J Cancer 2019;120:746-53.

26. Higuchi T, Uhlmann F. Cell cycle: passenger acrobatics. Nature 2003;426:780-1.

27. Liu J, Meng H, Li S, et al. Identification of Potential Biomarkers in Association With Pro-gression and Prognosis in Epithelial Ovarian Cancer by Integrated Bioinformatics Anal-ysis. Front Genet 2019;10:1031.

28. Itzel T, Scholz P, Maass T, et al. Translating bioinformatics in oncology: guilt-by-profiling analysis and identification of KIF18B and CDCA3 as novel driver genes in carcinogenesis. Bioinformatics 2015;31:216-24.

29. Kaneko N, Miura K, Gu Z, et al. siRNA-mediated knockdown against CDCA1 and KNTC2, both frequently overexpressed in colorectal and gastric cancers, suppresses cell prolif-eration and induces apoptosis. Biochem Biophys Res Commun 2009;390:1235-40.

30. Sawyer E, Roylance R, Petridis C, et al. Genetic predisposition to in situ and invasive lobular carcinoma of the breast. PLoS Genet 2014;10:e1004285.

31. Yoshihara K, Tajima A, Komata D, et al. Gene expression profiling of advanced-stage serous ovarian cancers distinguishes novel subclasses and implicates ZEB2 in tumor progression and prognosis. Cancer Sci 2009;100:1421-8.

32. Lu KH, Patterson AP, Wang L, et al. Selection of potential markers for epithelial ovarian cancer with gene expression arrays and recursive descent partition analysis. Clin Cancer Res 2004;10:3291-300.

33. Bonome T, Levine DA, Shih J, et al. A gene signature predicting for survival in subop-timally debulked patients 
with ovarian cancer. Cancer Res 2008;68:5478-86.

34. Wright JD, Hou JY, Burke WM, et al. Utilization and Toxicity of Alternative Delivery Methods of Adjuvant Chemotherapy for Ovarian Cancer. Obstet Gynecol 2016;127:985-91.

35. Markman M. Management of ovarian cancer. An impressive history of improvement in survival and quality of life. Oncology (Williston Park) 2006;20:347-354; discussion 354, 357-348, 364 passim.

36. Qian J, Lesage B, Beullens M, et al. PP1/Repoman dephosphorylates mitotic histone $\mathrm{H} 3$ at $\mathrm{T} 3$ and regulates chromosomal aurora $\mathrm{B}$ targeting. Curr Biol 2011;21:766-73.

37. Hu Q, Fu J, Luo B, et al. OY-TES-1 may regulate the malignant behavior of liver cancer via NANOG, CD9, CCND2 and CDCA3: a bioinformatic analysis combine with RNAi and oligonucleotide microarray. Oncol Rep 2015;33:1965-75.

38. Uchida F, Uzawa K, Kasamatsu A, et al. Overexpression of cell cycle regulator CDCA3 promotes oral cancer progression by enhancing cell proliferation with prevention of G1 phase arrest. BMC Cancer 2012;12:321.

39. Watanabe-Fukunaga R, Iida S, Shimizu Y, et al. SEI family of nuclear factors regulates p53-dependent transcriptional activation. Genes Cells 2005;10:851-60.

40. Chang IW, Lin VC, He HL, et al. CDCA5 overexpression is an indicator of poor prognosis in patients with urothelial carcinomas of the upper urinary tract and urinary bladder. Am J Transl Res 2015;7:710-22.

41. Cheng WY, Ou Yang TH, Anastassiou D. Development of a prognostic model for breast cancer survival in an open challenge environment. Sci Transl Med 2013;5:181ra50.

42. Ho DW, Kai AK, Ng IO. TCGA whole-transcriptome sequencing data reveals significantly dysregulated genes and signaling pathways in hepatocellular carcinoma. Front Med 2015;9:322-30.

43. Dai C, Miao CX, Xu XM, et al. Transcriptional activation of human CDCA8 gene regulated by transcription factor NF-Y in embryonic stem cells and cancer cells. J Biol Chem 2015;290:22423-34.

Cite this article as: Chen C, Chen S, Luo M, Yan H, Pang L, Zhu C, Tan W, Zhao Q, Lai J, Li H. The role of the CDCA gene family in ovarian cancer. Ann Transl Med 2020;8(5):190. doi: 10.21037/atm.2020.01.99
44. Hayama S, Daigo Y, Yamabuki T, et al. Phosphorylation and activation of cell division cycle associated 8 by aurora kinase B plays a significant role in human lung carcinogenesis. Cancer Res 2007;67:4113-22.

45. Jiao DC, Lu ZD, Qiao JH et al. Expression of CDCA8 correlates closely with FOXM1 in breast cancer: public microarray data analysis and immunohistochemical study. Neoplasma 2015;62:464-9.

46. Van den Bossche J, Deben C, Op de Beeck K, et al. Towards Prognostic Profiling of Non-Small Cell Lung Cancer: New Perspectives on the Relevance of PoloLike Kinase 1 Expression, the TP53 Mutation Status and Hypoxia. J Cancer 2017;8:1441-52.

47. Bowles DW, Diamond JR, Lam ET, et al. Phase I study of oral rigosertib (ON 01910.Na), a dual inhibitor of the $\mathrm{PI} 3 \mathrm{~K}$ and Plk1 pathways, in adult patients with advanced solid ma-lignancies. Clin Cancer Res 2014;20:1656-65.

48. Deng H, Jiang Q, Yang Y, et al. Intravenous liposomal delivery of the short hairpin RNAs against Plk1 controls the growth of established human hepatocellular carcinoma. Cancer Biol Ther 2011;11:401-9.

49. Li R, Jiang X, Zhang Y, et al. Cyclin B2 Overexpression in Human Hepatocellular Carci-noma is Associated with Poor Prognosis. Arch Med Res 2019;50:10-7.

50. Zhang R, Wei RL, Du W, et al. Long noncoding RNA ENST00000413528 sponges mi-croRNA-593-5p to modulate human glioma growth via polo-like kinase 1 . CNS Neurosci Ther 2019;25:842-54.

51. Prat J. New insights into ovarian cancer pathology. Ann Oncol 2012;23 Suppl 10:x111-117.

52. McCluggage WG. Morphological subtypes of ovarian carcinoma: a review with emphasis on new developments and pathogenesis. Pathology 2011;43:420-32.

53. Köbel M, Kalloger SE, Huntsman DG et al. Differences in tumor type in low-stage versus high-stage ovarian carcinomas. Int J Gynecol Pathol 2010;29:203-11.

54. Seidman JD, Horkayne-Szakaly I, Haiba M, et al. The histologic type and stage distribu-tion of ovarian carcinomas of surface epithelial origin. Int J Gynecol Pathol 2004;23:41-4. 


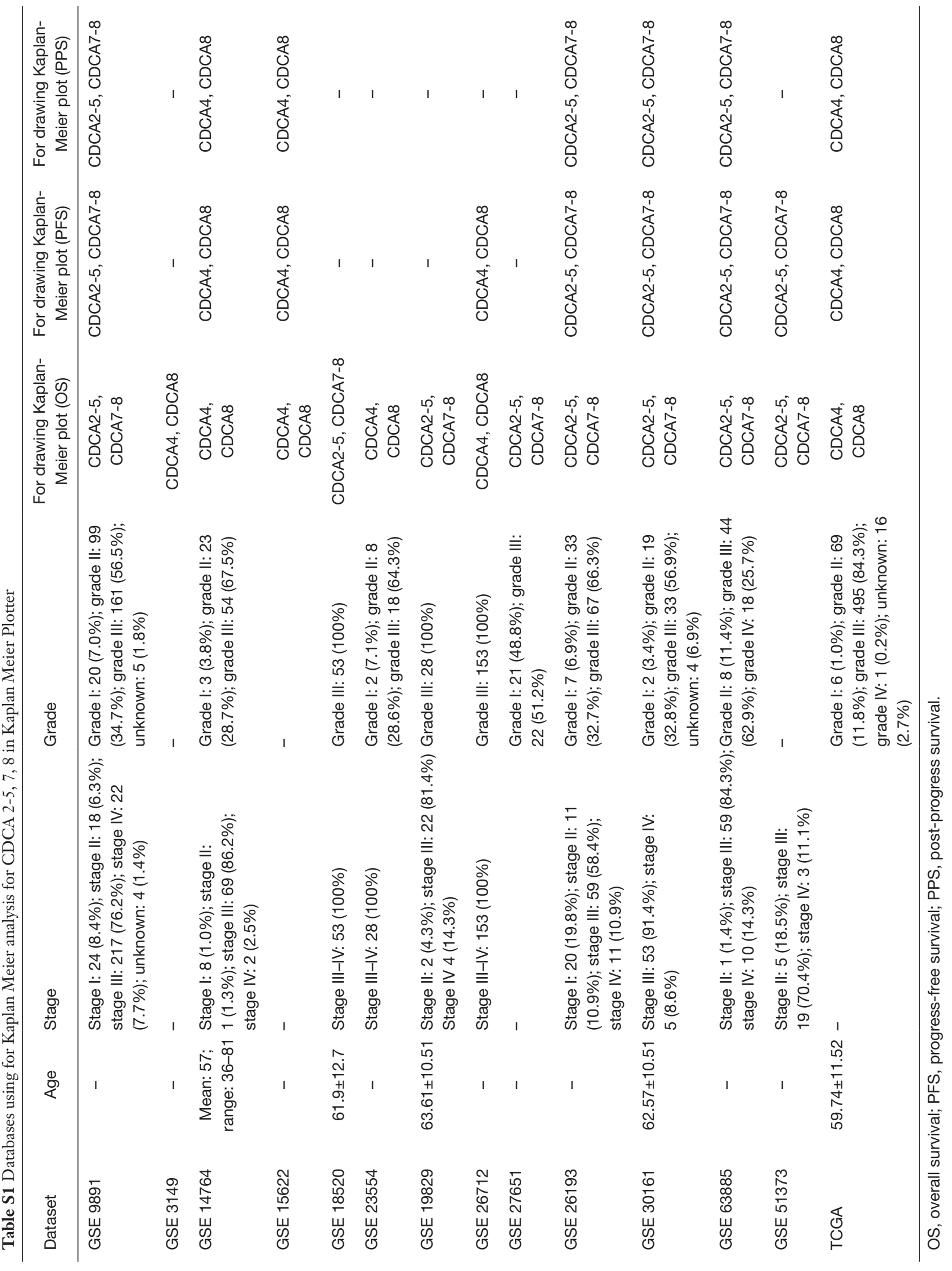


Table S2 Univariate and Multivariate analysis of risk factors for overall survival

\begin{tabular}{|c|c|c|c|c|}
\hline Factors & \multicolumn{2}{|c|}{ Univariate analysis } & \multicolumn{2}{|c|}{ Multivariate analysis } \\
\hline Grade & $1.46(1.15-1.85)$ & 0.002 & & 0.333 \\
\hline Stage & $1.963(1.548-2.490)$ & $2.70 \mathrm{E}-08$ & $2.054(1.587-2.658)$ & $4.4 \mathrm{E}-08$ \\
\hline CDCA2 & 1.089 (1.013-1.172) & 0.021 & & 0.952 \\
\hline CDCA4 & $1.094(1.014-1.181)$ & 0.021 & & 0.807 \\
\hline CDCA5 & $1.076(1.010-1.147)$ & 0.024 & & 0.279 \\
\hline CDCA7 & 1.075 (1.002-1.153) & 0.044 & $1.215(1.007-1.467)$ & 0.042 \\
\hline CDCA8 & $1.062(1.001-1.127)$ & 0.045 & & 0.714 \\
\hline
\end{tabular}

GSE 18920, GSE 26193, GSE 30161, GSE 63888 were used for analysis.

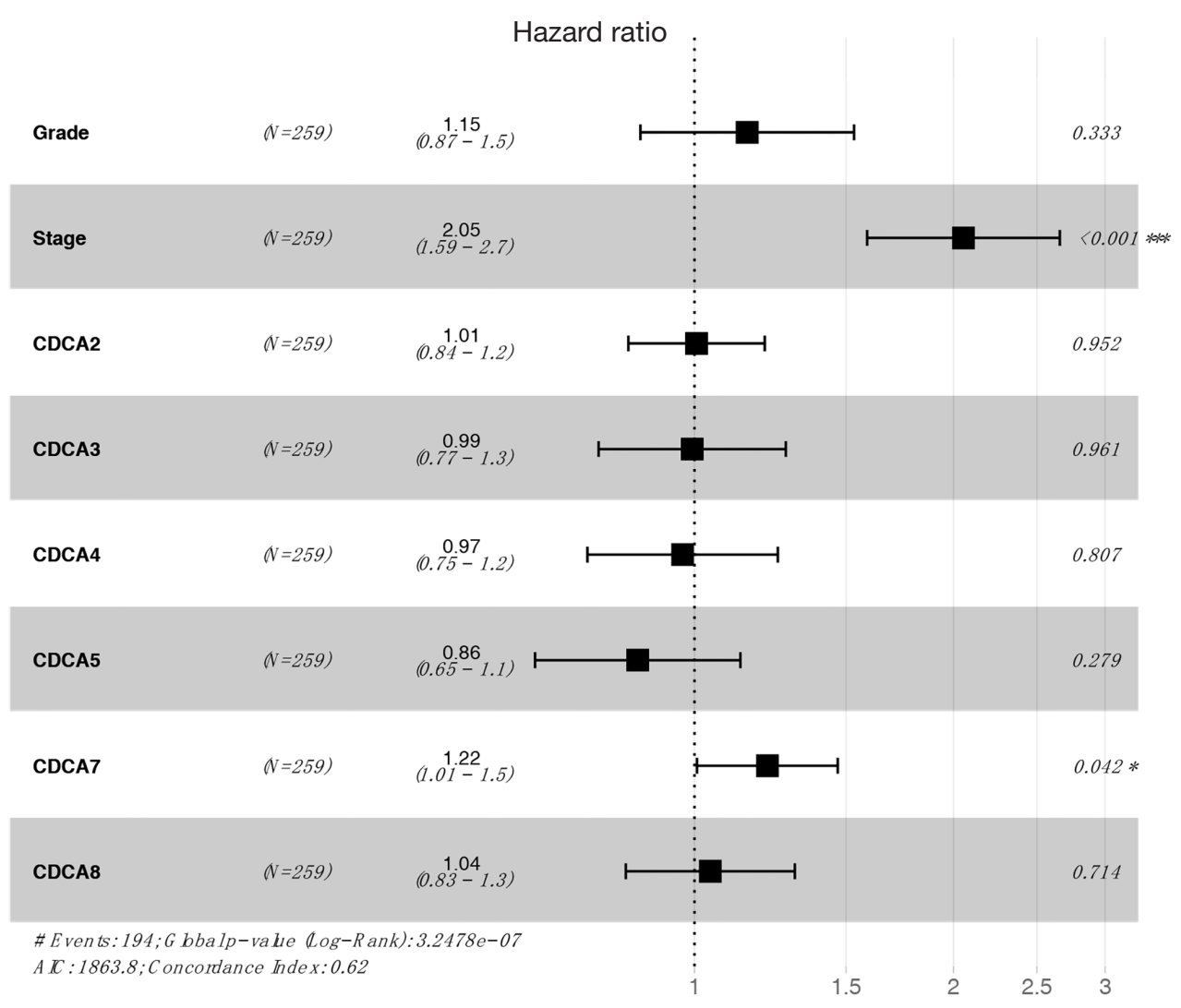

Figure S1 The multivariate analysis for risk factors and CDCA family genes. 